\title{
Conjoint alcohol and tobacco use among tuberculosis patients in public primary healthcare in South Africa
}

\author{
K Peltzer, ${ }^{1-3} \mathrm{PhD}$ \\ ${ }^{1}$ HIV/AIDS, STI and TB (HAST) Research Programme, Human Sciences Research Council, Pretoria, South Africa \\ ${ }^{2}$ Department of Psychology, University of Limpopo, Turfloop, South Africa \\ ${ }^{3}$ ASEAN Institute for Health Development, Madidol University, Salaya, Phutthamonthon, Nakhonpathom, Thailand
}

Corresponding author: K Peltzer (kpeltzer@hrsc.ac.za)

\begin{abstract}
Objective. To determine the prevalence of, and factors associated with conjoint alcohol and tobacco use among tuberculosis (TB) patients in South Africa (SA).

Methods. In a cross-sectional survey, 4900 (54.5\% men, 45.5\% women) consecutively selected TB patients (including new TB and new TB retreatment patients) from 42 public primary care clinics in three districts in SA were assessed using various measures (including those for alcohol and tobacco use), within one month of anti-TB treatment.

Results. Overall, 10.1\% (15.5\% among men; 3.4\% among women) were conjointly hazardous, harmful or dependent alcohol users and daily or almost-daily tobacco users. The proportion of daily or almost-daily tobacco users among hazardous, harmful or dependent alcohol users was 48.9\%, (53.3\% among men; $26.4 \%$ among women). Those with hazardous, harmful or dependent alcohol use had significantly higher odds of having anxiety and/or depression (odds ratio (OR) 1.37; confidence interval (CI) 1.13 - 1.65) and exhibiting daily or almost-daily tobacco use (OR 5.94; CI 4.33 - 5.87). The mean \pm standard deviation alcohol use disorders identification test (AUDIT) score among conjoint hazardous, harmful or dependent alcohol users and daily or almost-daily tobacco users was significantly higher (17.1 \pm 6.1$)$ than among hazardous, harmful or dependent alcohol users who were not current tobacco users $(15.4 \pm 5.6)(p<0.001)$. In multivariate analysis, male gender, coloured ethnicity, lower education and greater poverty, TB retreatment patient status and non-adherence to anti-TB medication were associated with a greater risk for conjoint alcohol and tobacco use.

Conclusions. A high prevalence and several risk factors for conjoint alcohol and tobacco use were found among TB patients. The findings of this study call for dual-intervention approaches to alcohol and tobacco use.
\end{abstract}

S Afr J Psych 2014;20(1):21-26. DOI:10.7196/SAJP.482

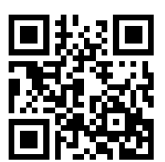

Cigarette smoking has consistently been associated with an increased risk of Mycobacterium tuberculosis infection, subsequent disease development and poor treatment outcomes. ${ }^{[1,2]}$ Alcohol misuse also augments the likelihood of developing tuberculosis (TB) and has been associated with poor treatment adherence and a significantly amplified risk of relapse and death during and after TB treatment. ${ }^{[1,3,4]} \mathrm{A}$ study in China found that smoking alone or sole alcohol consumption had no relationship with $\mathrm{TB}$, but smoking together with alcohol abuse was probably a risk factor for pulmonary $\mathrm{TB} .{ }^{[5]}$

There is a high degree of association between alcohol and nicotine dependence. ${ }^{[6-8]}$ Compared with one particular dependence, associated nicotine and alcohol dependence is more severe and the course is more unfavourable. ${ }^{[6]}$ According to Littleton et al. ${ }^{[9]}$ the 'major reasons are that concurrent alcohol use, and/or prior alcohol exposure, may change the reinforcing effects of nicotine, and that each drug becomes a pharmacological cue for the expectation of the other. If so, then smokers whose nicotine dependence is impacted by alcohol, represent a large and distinct sub-population in which both the therapeutic and molecular targets for smoking cessation are altered.'

In general, people with a substance use disorder also have higher comorbid rates of mental disorders than vice versa,${ }^{[7,8,10]}$ possibly inclu- ding post-traumatic stress disorder (PTSD). ${ }^{[11]}$ Nicotine dependence has also been associated with suicide attempts, ${ }^{[12]}$ and poor quality of life has been associated with psychoactive substance use. ${ }^{[13]}$ Conjoint alcohol and tobacco use has been associated with lower education, ${ }^{[14]}$ female gender, ${ }^{[14]}$ younger age ${ }^{[15]}$ and mental problems including depression and anxiety disorders. ${ }^{[1,16,17]}$ There is a dearth of studies investigating conjoint alcohol and tobacco use among TB patients. Therefore, congruent with the National Mental Health Action Plan, ${ }^{[18]}$ the objective of this study was to explore the prevalence of conjoint alcohol and tobacco use (v. alcohol or tobacco use) and to determine factors (including mental disorders) associated with such use among TB patients in South Africa (SA).

\section{Methods}

\section{Study design, sample and procedure}

This was a cross-sectional survey with TB patients in primary care clinics in SA. Three provinces with some of the highest TB caseloads were selected, and one district with the highest TB caseload in each province $(n=3)$ was ultimately included in the study: ${ }^{[19]}$ Siyanda in the Northern Cape, Nelson Mandela Metropole in the Eastern Cape, and eThekwini in KwaZulu-Natal. Within each study district, 14 primary healthcare facilities were selected on the basis of the 
highest TB caseload per clinic $(n=42)$. Health facilities were primary healthcare clinics or community health centres. All new TB and new TB retreatment patients were consecutively interviewed within one month of commencing treatment. Interviews were conducted by trained external research assistants for a period of 6 months from May to October 2011. Healthcare providers identified eligible patients aged $\geq 18$ years, informed them about the study and referred them for participation if interested. A research assistant asked for patient consent to participation for patients attending the primary care facility. Ethical approval was granted by the Human Sciences Research Council (HSRC) Research Ethics Committee (protocol REC no. 1/16/02/11) and the National Department of Health (NDoH).

\section{Measures \\ Alcohol consumption}

The 10-item alcohol use disorders identification test (AUDIT) ${ }^{[19]}$ is used to assess level of alcohol consumption ( 3 items), symptoms of alcohol dependence ( 3 items) and problems associated with alcoho use (4 items). Responses are rated on a 5-point Likert scale (from 0 to 4; maximum score 40). Higher AUDIT scores indicate more severe levels of risk: $\geq 8$ indicates a tendency for problematic drinking and $\geq 20$ indicates probable alcohol dependence. The AUDIT has been validated in HIV-positive patients in SA, with excellent sensitivity and specificity in detecting mini international neuropsychiatric interview (MINI)-defined dependence or abuse (area under the receiver-operating characteristic curve 0.96$),{ }^{[21]}$ and among TB/HIVpositive patients in primary care in Zambia, demonstrating good discriminatory ability in detecting MINI-defined current alcohol use disorders (AUDIT score: 0.98 in women; 0.75 in men) ${ }^{[22]}$ Cronbach's a for the AUDIT in this sample was 0.92, indicating excellent reliability.

\section{Tobacco use}

Two questions were asked about the use of tobacco products: (i) Do you currently use one or more of the following tobacco products (cigarettes, snuff, chewing tobacco, cigars, etc.)? - response options were 'yes' and 'no'; (ii) During the past month, how often have you used one or more of the following tobacco products (cigarettes, snuff, chewing tobacco, cigars, etc.) - response options were 'once or twice', 'weekly', 'almost daily' and 'daily.'[23]

\section{Social functioning (SF-12)}

The SF-12 is a measure of general health functioning, with 12 items reflecting the following 8 subdomains: self-perceived general health ( 1 item), bodily pain ( 1 item), physical functioning ( 2 items), physical role (2 items), vitality ( 1 item), general health $(1$ item), social functioning ( 1 item), mental health ( 2 items) and emotional role (2 items). Cronbach's a for the SF-12 was 0.80 in this study. For each respondent, the SF-12 scoring algorithm generates a physical health component summary (PCS)-12 score and a mental health component summary (MCS)-12 score. These scores are created by weighting and then summing the SF-12 item responses using two separate sets of weights (a physical weight and a mental weight) and then normalising the weighted sums to be comparable with a population mean \pm standard deviation (SD) score of $50 \pm 10 .{ }^{[24]}$ The lower the physical (PCS-12) or mental health score (MCS-12), the more activity limitations a person has. By taking the median of the PCS and MCS as a cut-off point, the PCS and MCS were dichotomised as poor $=0$ or $\operatorname{good}=1$.

\section{Kessler psychological distress scale (K-10)}

The $\mathrm{K}-10$ was used to measure global psychological distress, including significant pathology which does not meet formal criteria for a psychiatric illness. ${ }^{[25,26]}$ The K-10 has been shown to capture variability related to non-specific depression, anxiety and substance abuse, but does not measure suicidality or psychoses. ${ }^{[27]}$ The scale serves to identify individuals who are likely to meet formal definitions for anxiety and/or depressive disorders, as well as to identify individuals with sub-clinical illness who may not meet formal definitions for a specific disorder. ${ }^{[25]}$ The K-10 has demonstrated moderate discriminatory ability in detecting depression and anxiety disorders in the general population in SA (area under the receiver-operating curves of 0.73 and 0.72 , respectively; cut-off 16 ). ${ }^{[28]}$ The scale was used in this study as a binary variable comparing scores of $10-15 \mathrm{v}$. $\geq 16$. The internal reliability coefficient for the K-10 was $\alpha=0.92$.

\section{Primary care PTSD screen (PC-PTSD) ${ }^{[29]}$}

The four items on the PC-PTSD correspond to the four factors (i.e. re-experiencing, avoidance, hyperarousal and numbing) specific to the PTSD construct. A cutoff of 2 was used for PTSD-positive screening. ${ }^{[30,31]}$ Cronbach's $\alpha$ for the PC-PTSD in this study was 0.89 .

\section{Suicidal behaviour}

This was assessed with three questions; two assessing suicidal ideation (ever seriously thought about committing suicide and ever made a plan for committing suicide) and one assessing whether respondents had ever attempted suicide. Response options were 'yes' and 'no'.

\section{Socioeconomic characteristics}

Age, gender, educational level, marital status, income, employment status and residential status were assessed. Using a previously used measure, ${ }^{[32]}$ poverty was assessed with five items based on the availability or non-availability of shelter, fuel or electricity, clean water, food and cash income in the past week. Response options ranged from $1=$ 'not one day' to 4 = 'every day of the week'. Poverty was defined as a higher score on non-availability of essential items. The total score ranged from 5 to 20; 5 being low, 6 - 12 medium and 13 - 20 high poverty. Cronbach's $\alpha$ for the poverty index was 0.89 in this sample.

TB treatment status, HIV status and antiretroviral therapy (ART) were assessed by self-report and medical information. Non-adherence to anti-TB medication was assessed with the question: 'In your TB treatment in the past 3 - 4 weeks, what percentage of your anti-TB medication were your taking?'. Using the visual analogue scale, nonadherence to TB medication was defined as having taken $<90 \%$ of the prescribed anti-TB medication.

\section{Data analysis}

Data were analysed using SPSS (version 19.0). Frequencies and means $( \pm$ SDs) were calculated to describe the sample. Associations with hazardous, harmful or dependent alcohol use and daily or almost-daily tobacco use, and hazardous, harmful or dependent 
alcohol use or daily or almost-daily tobacco use (without dual alcohol and tobacco use) were identified using logistic regression analyses. Following each univariate regression, multivariate regression models were constructed. Independent variables from the univariate analyses were entered into the multivariate model if significant at $p<0.05$. For each model, $R^{2}$ values were presented to describe the amount of variance explained by the multivariate model. A $p$-value $<0.05$ was regarded as statistically significant.

Table 1. Sample characteristics

\begin{tabular}{|c|c|c|c|}
\hline Sociodemographic characteristics & $\begin{array}{l}\text { Total } \\
n(\%)\end{array}$ & $\begin{array}{l}\text { Hazardous, harmful or } \\
\text { dependent alcohol use } \\
\text { AND daily or almost-daily } \\
\text { tobacco use } \\
n(\%)\end{array}$ & $\begin{array}{l}\text { Hazardous, harmful or } \\
\text { dependent alcohol use } \\
\text { OR daily or almost-daily } \\
\text { tobacco use } \\
n(\%)\end{array}$ \\
\hline All & $4900(100)$ & $485(10.1)$ & $1122(26.0)$ \\
\hline \multicolumn{4}{|l|}{ Gender } \\
\hline Male & $2631(54.5)$ & $401(15.5)$ & $789(36.2)$ \\
\hline Female & $2194(45.5)$ & $74(3.4)$ & $317(15.2)$ \\
\hline \multicolumn{4}{|l|}{ Age (years) } \\
\hline $18-30$ & $1769(36.6)$ & $152(8.7)$ & $346(21.7)$ \\
\hline $31-44$ & $2078(41.8)$ & $182(9.2)$ & $489(27.3)$ \\
\hline$\geq 45$ & $1040(21.5)$ & $142(13.9)$ & $270(30.7)$ \\
\hline \multicolumn{4}{|l|}{ Marital status } \\
\hline Never married & $3323(72.7)$ & $321(9.7)$ & $752(25.2)$ \\
\hline Married/cohabitating & $982(21.5)$ & $106(10.8)$ & $243(27.6)$ \\
\hline Separated/divorced/widowed & $265(5.8)$ & $33(12.1)$ & $70(29.3)$ \\
\hline \multicolumn{4}{|l|}{ Education } \\
\hline$\leq$ Grade 7 & $1269(26.3)$ & $179(14.3)$ & $348(32.4)$ \\
\hline Grade 8 - 11 & $2213(45.9)$ & $227(10.4)$ & $533(27.3)$ \\
\hline$\geq$ Grade 12 & $1336(27.7)$ & $74(5.6)$ & $225(18.1)$ \\
\hline \multicolumn{4}{|l|}{ Poverty index (range 5 - 20) } \\
\hline Low (5) & $1592(35.0)$ & $124(7.7)$ & $321(21.7)$ \\
\hline Medium (6 - 12) & $2195(48.2)$ & $211(9.6)$ & $515(26.0)$ \\
\hline High $(13-20)$ & $768(16.9)$ & $110(4.4)$ & $220(33.7)$ \\
\hline \multicolumn{4}{|l|}{ Population group } \\
\hline Black & $4078(84.6)$ & $354(8.8)$ & $861(23.4)$ \\
\hline Coloured ${ }^{*}$ & $634(13.1)$ & $115(18.6)$ & $222(43.8)$ \\
\hline Indian/Asian/white/other & $114(2.3)$ & $9(8.2)$ & $25(24.8)$ \\
\hline \multicolumn{4}{|l|}{ Health characteristics } \\
\hline Physical health (PCS) & $2182(53.8)$ & $218(10.1)$ & $495(25.4)$ \\
\hline Mental health (MCS) & $1969(48.6)$ & $186(9.6)$ & $421(23.9)$ \\
\hline New TB patient & $3650(76.6)$ & $322(8.8)$ & $827(24.8)$ \\
\hline $\mathrm{TB}$ retreatment patient & $1113(23.4)$ & $160(14.4)$ & $285(30.7)$ \\
\hline HIV-positive & $2585(59.9)$ & $209(8.1)$ & $547(23.0)$ \\
\hline HIV-negative & $1728(40.1)$ & $208(12.0)$ & $446(29.2)$ \\
\hline HIV status unknown & $385(9.6)$ & $55(14.5)$ & $110(34.3)$ \\
\hline Non-adherence to TB treatment & $1138(33.9)$ & $158(14.0)$ & $297(30.6)$ \\
\hline \multicolumn{4}{|l|}{ Mental disorder } \\
\hline Anxiety and depression $(\mathrm{K}-10>15)$ & $3913(81.1)$ & $403(10.4)$ & $922(26.5)$ \\
\hline PTSD & $1441(29.4)$ & $160(11.3)$ & $350(27.8)$ \\
\hline Suicide attempt & $131(3.1)$ & $23(18.3)$ & $31(30.1)$ \\
\hline
\end{tabular}




\section{ARTICLE}

\section{Results}

Overall, $10.1 \%$ of TB patients (15.5\% among men; $3.4 \%$ among women) displayed conjoint hazardous, harmful or dependent alcohol use and daily or almost-daily tobacco use, and 26.0\% (36.2\% among men; $15.2 \%$ among women) were hazardous, harmful or dependent alcohol users or daily or almost-daily tobacco users (Table 1).

The proportion of hazardous, harmful or dependent alcohol users was $23.3 \%$ (31.8\% among men; $13.0 \%$ among women), and the proportion of daily or almost-daily tobacco users was $20 \%(29.5 \%$ among men; $8.5 \%$ among women). The proportion of daily or almostdaily tobacco users among hazardous, harmful or dependent alcohol users was $48.9 \%$ (53.3\% among men; $26.4 \%$ among women). The burden of hazardous, harmful or dependent alcohol users and/or daily or almost-daily tobacco users was $33.4 \%$ (46.1\% among men; $18.1 \%$ among women). The conjoint use of alcohol and tobacco was comorbid with anxiety/depression in $8.4 \%$ of the sample $(12.9 \%$ among men; 2.8\% among women) (Table 2). Those with hazardous, harmful or dependent alcohol use had significantly higher odds of having anxiety and/or depression (odds ratio (OR) 1.37; confidence interval (CI) $1.13-1.65$ ) and of exhibiting daily or almost-daily tobacco use (OR 5.94; CI 4.33 - 5.87). The mean \pm SD AUDIT score among conjoint hazardous, harmful or dependent alcohol users and daily or almost-daily tobacco users was significantly higher (17.1 \pm 6.1$)$ than among hazardous, harmful or dependent alcohol users who were not current tobacco users $(15.4 \pm 5.6)(p<0.001)$.

\section{Associations with alcohol and/or tobacco use}

In multivariate analysis, male gender, coloured ethnicity, a lower level of education and greater poverty were found to be associated with conjoint alcohol and tobacco use, as well as with alcohol or tobacco use. In relation to health characteristics, TB retreatment patient status and non-adherence to anti-TB medication were associated with conjoint alcohol and tobacco use upon multivariate analysis, with non-adherence also associated with alcohol or tobacco use. In bivariate analysis, in terms of mental problems, having ever attempted suicide was associated with conjoint alcohol and tobacco use. Anxiety/ depression and PTSD were neither associated with conjoint alcohol and tobacco use, nor with alcohol or tobacco use (Table 3).

\section{Discussion}

This study revealed a high prevalence of conjoint alcohol and tobacco use in a large sample of TB patients in public primary care in SA. In agreement with other studies, ${ }^{[6]}$ conjoint alcohol and tobacco users had significantly higher AUDIT scores than alcohol-only users. Similar rates of comorbidity between alcohol use disorders (hazardous, harmful or dependent alcohol use; AUDIT score $\geq 8$ ) and nicotine dependence (smoking daily) were reported among general hospital patients in Brazil (42.8\%). ${ }^{[33]}$ Importantly, the prevalence of conjoint alcohol and tobacco use in the cohort of TB patients described here may have been higher than in the general population of SA. ${ }^{[33]}$

Several risk factors (sociodemographic and health factors but not mental problems) were jointly associated with alcohol and tobacco use, as well as alcohol or tobacco use. The prevalence of conjoint alcohol and tobacco use was 6.16 times higher among male than female patients. This finding conforms with previous studies in SA, ${ }^{[34]}$ but is contrary to the results of a study conducted in Brazil. ${ }^{[33]}$ In agreement with other studies, ${ }^{[14]}$ a lower education level and greater degree of poverty were associated with conjoint alcohol and tobacco use. In terms of population groups, the coloured population had the highest risk for dual substance use, as has been reported in previous studies performed in SA. ${ }^{[34,35]}$ Furthermore, congruent with previous findings, ${ }^{[1-4]} \mathrm{TB}$ retreatment patient status and non-adherence to anti-TB medication were associated with conjoint alcohol and tobacco use. However, unlike in other studies, ${ }^{[7,8,10-17]}$ there was no association between mental problems, PTSD, poor quality of life and alcohol and tobacco use.

\section{Conclusion}

The survey revealed a high prevalence and several risk factors of conjoint alcohol and tobacco use among $\mathrm{TB}$ patients in the cohort described. These findings are of great importance, suggesting that conjoint or sequential interventions in $\mathrm{TB}$ patients who misuse alcohol and tobacco are appropriate and should be initiated in this public primary care setting. ${ }^{[14]}$ Adequate dual-intervention approaches for both alcohol and tobacco should assume strategies that include identifying sociodemographic and health risk factors and issues related to both substances. ${ }^{[14]}$

Table 2. Alcohol use disorder, nicotine dependence, anxiety/depression and overlap

\begin{tabular}{|c|c|c|c|c|}
\hline & $\begin{array}{l}\text { Total sample } \\
n(\%)\end{array}$ & $\begin{array}{l}\text { Men } \\
n(\%)\end{array}$ & $\begin{array}{l}\text { Women } \\
n(\%)\end{array}$ & $p$-value \\
\hline Hazardous, harmful or dependent alcohol users & $1120(23.3)$ & $820(31.8)$ & $280(13.0)$ & $<0.000$ \\
\hline Daily or almost-daily tobacco users & $980(20.0)$ & $777(29.5)$ & $187(8.5)$ & $<0.000$ \\
\hline $\begin{array}{l}\text { Proportion of current tobacco users among hazardous, harmful or dependent } \\
\text { alcohol users }\end{array}$ & $624(57.7)$ & $501(63.0)$ & $112(41.8)$ & $<0.000$ \\
\hline $\begin{array}{l}\text { Proportion of daily or almost-daily tobacco users among hazardous, harmful or } \\
\text { dependent alcohol users }\end{array}$ & $485(48.9)$ & $401(53.3)$ & $74(26.4)$ & $<0.000$ \\
\hline $\begin{array}{l}\text { Hazardous, harmful or dependent alcohol users and/or daily or almost-daily } \\
\text { tobacco users }\end{array}$ & $1607(33.4)$ & $1190(46.1)$ & $391(18.1)$ & $<0.000$ \\
\hline $\begin{array}{l}\text { Hazardous, harmful or dependent alcohol users and daily or almost-daily tobacco } \\
\text { users and anxiety/depression }\end{array}$ & $403(8.4)$ & $333(12.9)$ & $60(2.8)$ & $<0.000$ \\
\hline
\end{tabular}


Table 3. Association of socioeconomic and clinical characteristics, mental ill-health and alcohol and tobacco use among TB patients

\begin{tabular}{|c|c|c|c|c|}
\hline \multirow[b]{2}{*}{$\begin{array}{l}\text { Sociodemographic } \\
\text { characteristics }\end{array}$} & \multicolumn{2}{|c|}{$\begin{array}{l}\text { Hazardous, harmful or dependent alcohol use } \\
\text { AND daily or almost-daily tobacco use }\end{array}$} & \multicolumn{2}{|c|}{$\begin{array}{l}\text { Hazardous, harmful or dependent alcohol use } \\
\text { OR daily or almost-daily tobacco use }\end{array}$} \\
\hline & $\begin{array}{l}\text { Crude OR } \\
(95 \% \mathrm{CI})^{\star}\end{array}$ & $\begin{array}{l}\text { Adjusted OR } \\
(95 \% \mathrm{CI})^{\star, \dagger}\end{array}$ & $\begin{array}{l}\text { Crude OR } \\
(95 \% \mathrm{CI})^{\star}\end{array}$ & $\begin{array}{l}\text { Adjusted OR } \\
(95 \% \mathrm{CI})^{*, *}\end{array}$ \\
\hline \multicolumn{5}{|l|}{ Age (years) } \\
\hline $18-30$ & 1.00 & 1.00 & 1.00 & 1.00 \\
\hline $31-44$ & $1.07(0.85-1.34)$ & $1.01(0.71-1.39)$ & $1.30(1.13-1.49)^{\dagger \dagger}$ & $1.06(0.82-1.37)$ \\
\hline$\geq 45$ & $1.70(1.33-2.16)^{\dagger \dagger}$ & $1.07(0.78-1.52)$ & $1.70(1.44-2.00)^{\dagger \dagger}$ & $1.03(0.75-1.43)$ \\
\hline \multicolumn{5}{|l|}{ Gender } \\
\hline Female & 1.00 & 1.00 & 1.00 & 1.00 \\
\hline Male & $5.19(4.02-6.69)^{\dagger \dagger}$ & $6.16(4.13-9.19)^{\dagger \dagger}$ & $3.17(2.73-3.67)^{\dagger \dagger}$ & $3.23(2.57-4.06)^{\dagger \dagger}$ \\
\hline \multicolumn{5}{|l|}{ Population group } \\
\hline Black & 1.00 & 1.00 & 1.00 & 1.00 \\
\hline Coloured & $2.38(1.89-2.99)^{\dagger+}$ & $2.03(1.39-2.97)^{\dagger \dagger}$ & $2.55(2.10-3.09)^{\dagger \dagger}$ & $3.03(2.19-4.18)^{\dagger \dagger}$ \\
\hline Indian/Asian/other & $0.92(0.46-1.84)$ & $0.91(0.34-2.43)$ & $1.07(0.68-1.70)$ & $1.58(0.80-3.15)$ \\
\hline \multicolumn{5}{|l|}{ Marital status } \\
\hline Not married & 1.00 & - & 1.00 & - \\
\hline Married/cohabitating & $1.12(0.89-1.42)$ & & $1.14(0.96-1.35)$ & \\
\hline Separated/divorced/widowed & $1.29(0.88-1.88)$ & & $1.23(0.92-1.65)$ & \\
\hline \multicolumn{5}{|l|}{ Education } \\
\hline$\leq$ Grade 7 & 1.00 & 1.00 & 1.00 & 1.00 \\
\hline Grade 8 - 11 & $0.70(0.57-0.86)^{\dagger \dagger}$ & $0.68(0.49-0.95)^{\S}$ & $0.78(0.67-0.92)^{\star *}$ & $0.88(0.67-1.16)$ \\
\hline$\geq$ Grade 12 & $0.36(0.27-0.47)^{\dagger \dagger}$ & $0.29(0.17-0.48)^{\dagger \dagger}$ & $0.46(0.38-0.56)^{\dagger \dagger}$ & $0.51(0.37-0.71)^{\dagger \dagger}$ \\
\hline \multicolumn{5}{|l|}{ Poverty } \\
\hline Low & 1.00 & 1.00 & 1.00 & 1.00 \\
\hline Medium & $1.27(1.01-1.60)^{\varsigma}$ & $0.91(0.64-1.28)$ & $1.26(1.08-1.48)^{\star *}$ & $1.29(1.01-1.65)^{\S}$ \\
\hline High & $2.01(1.53-2.64)^{\dagger \dagger}$ & $1.62(1.06-2.47)^{5}$ & $1.84(1.50-2.25)^{\dagger \dagger}$ & $1.15(0.80-1.65)$ \\
\hline \multicolumn{5}{|l|}{ Health characteristics } \\
\hline Physical health (PCS) & $1.02(0.83-1.28)$ & - & $0.98(0.86-1.17)$ & - \\
\hline Mental health (MCS) & $0.92(0.75-1.13)$ & - & $0.85(0.74-0.97)^{\S}$ & $0.88(0.70-1.09)$ \\
\hline New TB patient & 1.00 & 1.00 & 1.00 & 1.00 \\
\hline Retreatment TB patient & $1.74(1.42-2.14)^{\dagger \dagger}$ & $1.64(1.20-2.25)^{\star \star}$ & $1.46(1.27-1.68)^{\dagger \dagger}$ & $1.09(0.85-1.41)$ \\
\hline TB/HIV co-infected & $0.65(0.53-0.79)^{\dagger \dagger}$ & $0.92(0.62-1.33)$ & $0.73(0.63-0.84)^{\dagger \dagger}$ & $0.86(0.62-1.18)$ \\
\hline \multicolumn{5}{|l|}{ Adherence } \\
\hline TB non-adherence & $2.58(1.57-4.25)^{\dagger \dagger}$ & $1.73(1.28-2.33)^{\dagger \dagger}$ & $1.55(1.30-1.84)^{\dagger \dagger}$ & $1.42(1.13-1.79)^{\star *}$ \\
\hline \multicolumn{5}{|l|}{ Mental disorder } \\
\hline Anxiety and depression & $1.08(0.89-1.32)$ & - & $1.16(0.97-1.38)$ & - \\
\hline PTSD & $1.20(0.98-1.46)$ & - & $1.14(0.99-1.32)$ & - \\
\hline Suicide attempt & $2.01(1.27-3.20)^{\star *}$ & $1.85(0.93-3.69)$ & $1.25(0.81-1.91)$ & - \\
\hline \multicolumn{5}{|c|}{$\begin{array}{l}\mathrm{OR}=\text { odds ratio; PCS = physical health component summary; } \mathrm{MCS}=\text { mental health component summary; } \mathrm{TB}=\text { tuberculosis; } \mathrm{PTSD}=\text { post-traumatic stress disorder. } \\
{ }^{*} \text { Using 'enter' LR selection of variables. }\end{array}$} \\
\hline \multicolumn{5}{|c|}{${ }^{\dagger}$ Hosmer and Lemeshow $\chi^{2} 5.18$; degrees of freedom (df) 8; $p=0.738$; Cox and Snell $R^{2} 0.09$; Nagelkerke $R^{2} 0.19$} \\
\hline \multicolumn{5}{|c|}{${ }^{\ddagger}$ Hosmer and Lemeshow $\chi^{2} 12.93$; df $8 ; p=0.114 ;$ Cox and Snell $R^{2} 0.10$; Nagelkerke $R^{2} 0.15$} \\
\hline${ }^{5} p<0.05 ;{ }^{* *} p<0.01 ;{ }^{+1} p<0.001$ & & & & \\
\hline
\end{tabular}

Acknowledgement. The $\mathrm{NDoH}$ funded this study through a tender awarded to the HSRC: 'NDOH: 21/2010-2011 Implementation and monitoring of Screening and Brief Intervention for alcohol use disorders among Tuberculosis patients'. 


\section{References}

1. Bates MN, Khalakdina A, Pai M, et al. Risk of tuberculosis from exposure to tobacco smoke A systematic review and meta-analysis. Arch Intern Med 2007;167:335-342. [http://dx.doi org/10.1001/archinte.167.4.335]

2. Marais BJ, Lönnroth K, Lawn SD, et al. Tuberculosis comorbidity with communicable and non-communicable diseases: Integrating health services and control efforts. Lancet Infect Dis 2013;13(5):436-448. [http://dx.doi.org/10.1016/S1473-3099(13)70015-X]

3. Lönnroth $\mathrm{K}$, Williams BG, Stadlin S, Jaramillo E, Dye C. Alcohol use as a risk facto for tuberculosis: A systematic review. BMC Public Health 2008;8:289. [http://dx.doi. org/10.1186/1471-2458-8-289

4. Rehm J, Samokhvalov AV, Neuman MG, et al. The association between alcohol use, alcohol use disorders and tuberculosis (TB). A systematic review. BMC Public Health 2009;9:450. [http:// dx.doi.org/10.1186/1471-2458-9-450]

5. Dong B, Ge N, Zhou Y. Smoking and alcohol consumption as risk factors of pulmonary tuberculosis in Chengdu: A matched case-control study. Hua Xi Yi Ke Da Xue Xue Bao 2001;32(1):104-106

6. Diehl A, Scherbaum N. Nicotine dependence as comorbidity of alcohol dependence epidemiology, etiology and therapy. Fortschr Neurol Psychiatr 2008;76(1):14-20. [http://dx.doi. org/10.1055/s-2007-980076

7. Subramaniam M, Abdin E, Vaingankar J, Phua AM, Tee J, Chong SA. Prevalence and correlates of alcohol use disorders in the Singapore Mental Health Survey. Addiction 2012;107(8):1443-1452. [http://dx.doi.org/10.1111/j.1360-0443.2012.03830.x]

8. Farrell M, Howes S, Bebbington P, et al. Nicotine, alcohol and drug dependence, and psychiatric comorbidity - results of a national household survey. Int Rev Psychiatry 2003;15(1-2):50-56. [http://dx.doi.org/10.1080/0954026021000045949]

9. Littleton J, Barron S, Prendergast M, Nixon SJ. Smoking kills (alcoholics)! shouldn't we do something about it? Alcohol Alcohol 2007;42(3):167-173. [http://dx.doi.org/10.1093/alcalc/ agm019]

10. Jané-Llopis E, Matytsina I. Mental health and alcohol, drugs and tobacco: A review of the comorbidity between mental disorders and the use of alcohol, tobacco and illicit drugs. Dru Alcohol Rev 2006;25(6):515-536. [http://dx.doi.org/10.1080/09595230600944461]

11. Breslau N, Davis GC, Schultz LR. Posttraumatic stress disorder and the incidence of nicotine, alcohol, and other drug disorders in persons who have experienced trauma. Arch Gen Psychiatry 2003;60(3):289-294

12. Yaworski D, Robinson J, Sareen J, Bolton JM. The relation between nicotine dependence and suicide attempts in the general population. Can J Psychiatry 2011;56(3):161-170.

13. Moreira Tde C, Figueiró LR, Fernandes S, et al. Quality of life of users of psychoactive substances, relatives, and non-users assessed using the WHOQOL-BREF. Cien Saude Colet 2013;18(7):1953 1962.

14. Horn K, Gao X, Williams J, Helmkamp J, Furbee M, Manley W. Conjoint smoking and drinking: A case for dual-substance intervention among young emergency department patients. Acad Emerg Med 2000;7(10):1126-1134

15. Falk DE, Yi HY, Hiller-Sturmhöfel S. An epidemiologic analysis of co-occurring alcohol an tobacco use and disorders: Findings from the National Epidemiologic Survey on Alcohol an Related Conditions. Alcohol Res Health 2006;29(3):162-171.

16. Hertling I, Ramskogler K, Dvorak A, et al. Craving and other characteristics of the comorbidity of alcohol and nicotine dependence. Eur Psychiatry 2005;20(5-6):442-450. [http://dx.doi. org/10.1016/j.eurpsy.2005.06.003
17. Kelly MM, Grant C, Cooper S, Cooney JL. Anxiety and smoking cessation outcomes in alcoholdependent smokers. Nicotine Tob Res 2013;15(2):364-375. [http://dx.doi.org/10.1093/ntr/nts132]

18. Janse van Rensburg ABR. Contributions from the South African Society of Psychiatrists (SASOP) to the National Mental Health Action Plan. South African Journal of Psychiatry 2013;19(4):205212. [http://dx.doi.org/10.7196/SAJP.501]

19. Department of Health. National TB crisis management plan. Pretoria: Department of Health 2010

20. Babor TF, Higgins-Biddle JC. Brief Intervention for Hazardous and Harmful Drinking: A Manual for Use in Primary Care. Geneva: World Health Organization, Department of Mental Health an Substance Dependence, 2001.

21. Myer L, Smit J, Roux LL, Parker S, Stein DJ, Seedat S. Common mental disorders amon HIV-infected individuals in South Africa: Prevalence, predictors, and validation of brie psychiatric rating scales. AIDS Patient Care STDs 2008;22:147-158. [http://dx.doi.org/10.1089/ apc.2007.0102

22. Chishinga N, Kinyanda E, Weiss HA, Patel V, Ayles H, Seedat S. Validation of brief screening tools for depressive and alcohol use disorders among TB and HIV patients in primary care in Zambia. BMC Psychiatry 2011;11:75. [http://dx.doi.org/10.1186/1471-244X-11-75]

23. World Health Organization. Guidelines for controlling and monitoring the tobacco epidemic Geneva: WHO, 1998.

24. Ware JE, Kosinski M, Keller SD. How to Score the SF-12 Physical and Mental Health Summar Scales. 2nd end. Boston: Health Institute, New England Medical Center, 1995.

25. Kessler R, Andrews G, Colpe LJ, et al. Short screening scales to monitor population prevalence and trends in nonspecific psychological distress. Psychol Med 2002;32:959-976.

26. Kessler RC, Barker PR, Colpe LJ, et al. Screening for serious mental illness in the genera population. Arch Gen Psychiatry 2003;60:184-189.

27. Brooks RT, Beard J, Steel Z. Factor structure and interpretation of the K10. Psychol Assess 2006;18:62-70.

28. Andersen LS, Grimsrud A, Myer L, Williams DR, Stein DJ, Seedat S. The psychometric propertie of the K10 and K6 scales in screening for mood and anxiety disorders in the South African Stress and Health study. Intern J Meth Psychiat Res 2011;20:215-223. [http://dx.doi.org/10.1002/ mpr.351]

29. Prins A, Ouimette P, Kimerling R, et al. The primary care PTSD screen (PC-PTSD): Development and operating characteristics. Prim Care Psychiatry 2003;9:9-14

30. Van Dam D, Ehring T, Vedel E, Emmelkamp PM. Validation of the Primary Care Posttraumatic Stress Disorder screening questionnaire (PC-PTSD) in civilian substance use disorder patients. J Subs Abuse Treat 2010;39:105-113. [http://dx.doi.org/10.1016/j.jsat.2010.05.005]

31. Ouimette P, Wade M, Prins A, Schohn M. Identifying PTSD in primary care: Comparison of the Primary Care-PTSD screen (PC-PTSD) and the General Health Questionnaire-12 (GHQ). J Anxiety Dis 2008;22:337-343.

32. South African National AIDS Council (SANAC). The HIV Epidemic in South Africa: What Do We Know and How Has It Changed? http://www.hsrc.ac.za (accessed 6 August 2011).

33. Botega NJ, Mitsuushi GN, Azevedo RC, et al. Depression, alcohol use disorders and nicotine dependence among patients at a general hospital. Rev Bras Psiquiatr 2010;32(3):250-256

34. Peltzer K, Davids A, Njuho P. Alcohol use and problem drinking in South Africa: Findings from a national population-based survey in 2008. Afr J Psychiatry 2011;14:30-37.

35. Peltzer K. Tobacco use trends among adolescents and adults in South Africa. J Psychol Afr 2008;18(2):339-346. 